\title{
Identification of Objects by Machines using RFID Technology Identify Objetcs in Internet of Things
}

\author{
Douae Salim, Larbi Setti, Abdellatif Elabderrahmani \\ Advanced Technology Laboratory, Abdelmalek Essaâdi University \\ Larache, Morocco \\ Douae.salim@gmail.com, slarbi@hotmail.com, elabderrahmani@yahoo.fr
}

\begin{abstract}
Embedded Systems and Internet of Things know recently a revolution in terms of innovation. With connectivity and networks, embedded systems are becoming more communicative, intelligent, and autonomous. They had the ability to calculate, process information, and perception. Digital connectivity to physical objects requires, firstly, the identification of objects, in order to be able to recognize each object in a unique way and to collect the data stored, ensuring security and confidentiality. In this paper we will examine the RFID technology (Radio-frequency Identification), which enables wireless interaction over certain frequency of RFID readers with a network system, to uniquely identify, track and capture informations at varying distances without the need of human interaction. Typically, RFID readers, which emits electromagnetic waves at a certain power, in order to activate the RFID tags, if it presents in the reader's transmission range. RFID tags respond to the reader's request by emitting radio waves back with the data stored the chip. Furthermore, we will explore Identification algorithm and communication techniques between reader and tags to avoid collision, whenever a large volume of tags must be read together, or perform a transmission in the same RF field at the same time. In addition, we will describe communications protocol between reader and the information system.
\end{abstract}

Keywords-Internet of things, embedded systems, RFID, Tags, reader, radio-frequency.

\section{Introduction}

Identify physical objects is the first step we should perform in Internet of Things, several identification technology are available, all of them are line-of-sight, they require a close distance between reader and tag. In the other hand, RFID (Radio Frequency Identification)does not need to be very close to the objects, it is an ubiquitous automatic technology to identify and collect objects through radio frequency digital signals. The RF wave is typically electromagnetic, at least in the far field. The RF wave can also be predominantly electric or magnetic in the near field.With RFID we can detect, track, and know some specific informations about objects, it is used for many fields such as transport, health, home automation, Library, security, environment, industry.

\section{RFID Physical Components:}

The RFID system Composed of four main elements RFID tags, RFID readers, the air interface which define communication protocol, and edge servers. Typically, RFID readers emit radio-frequency signal that RFID 
tags would detect if present in the reader's transmission range, RFID tags respond to the reader's queries by emitting radio waves back with data stored in the chip.[1] Tags can be either passive or active. A passive tag has no physical power source. It harvests energy from the reader's generated radio waves, using back scattering modulation; and consumes that energy in carrying out processing and communication tasks.An active tag has a power source and may possess certain sensing capabilities for temperature or pressure.[2]

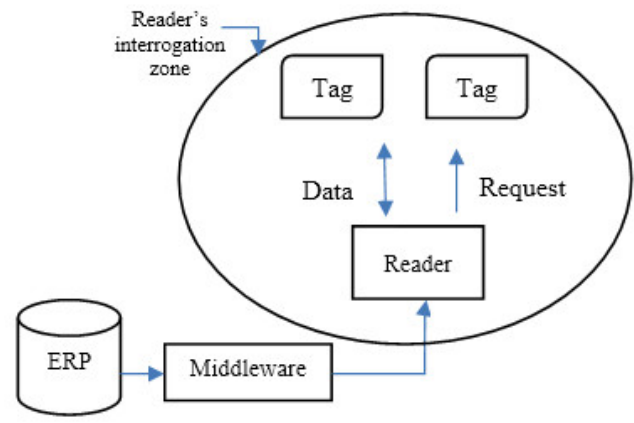

Figure. 1. Architecture of RFID System

Every tag has a unique identification code (ID). The length of ID may be different in different RFID standards; the tags in EPC class1 gen2 have 96 bit ID, but those of ISO-18000-6B (FDID, 2003) are 64 bit ID[3].It consists of a microchip programmed with information about a product and a coupling element an antenna. As schowing in figure 1 the architecture of RFID tag is constituted by modulation / demodulation bloc, a local memory containing information about product stored in data base, and a micro-controller that represent the intelligent part of tag.

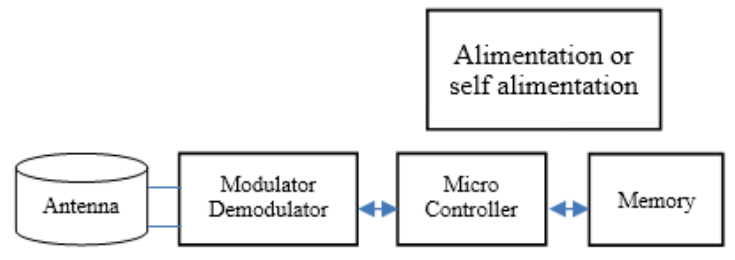

Figure. 2 . Architecture of RFID tag

Depending on the application and technology used, some interrogators not only read, but also remotely write to, the tags. For the majority of low cost tags, the power to activate the tag microchip is supplied by the reader through the tag antenna when the tag is in the interrogation zone of the reader.

Generally, the reader is constituted by (figure 2):

- An analog part regrouping:

- A local oscillator accorded on the frequency of a transmitted signal.

- A modulator/demodulator to transmit or to receive the numeric messages.

- An amplifier adapted on the antenna of emission / reception.

- A numeric part regrouping: 
- A micro-controller for the management of communication protocols, collisions...

- A communication interface.

- A local memory.[4]

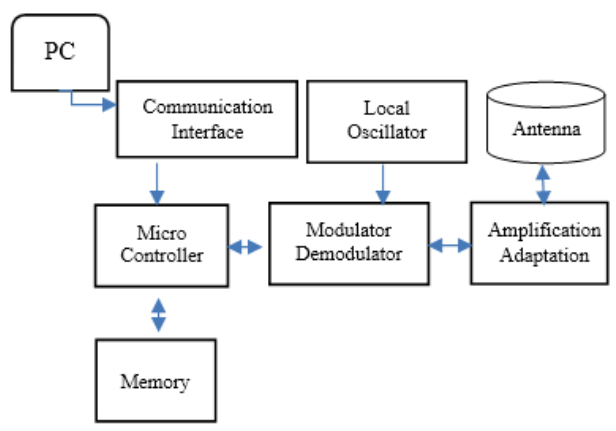

Figure . 2. Architecture of RFID integrator

In order for the chip to communicate with the reader, the tag must be open and recognized by the reader (same frequency, same speed, same modulation ...)

\section{RFID Interface}

The middleware RFID is the brain of the system. It manages the different readers and determines the interaction that they must have with the RFID tags. It is also the element that allows pre-processing of the captured information (filtering, routing, duplicate elimination, etc.) in order to facilitate their insertion in the databases of the information system.

\section{RFID Coupling}

Reader and tag communication depends on the coupling method. In RFID systems there are two type of coupling, capacitive and inductive, that allow to determine the read range and frequency of the system. The systems that use capacitive coupling use electric currents instead of the magnetic field in order to couple.

\begin{tabular}{|c|c|c|}
\hline Close Coupling & $\begin{array}{c}\text { Magnetic and } \\
\text { capacitive } \\
\text { coupling }\end{array}$ & $\begin{array}{c}\text { Inductive } \\
\text { coupling }\end{array}$ \\
\hline -Range $\sim 1 \mathrm{~cm}$ & -Range $1 \mathrm{~cm}$ & -Long range \\
$-\mathrm{LF}-125 \mathrm{kHz}$ & to $1 \mathrm{~m}$ & -Range $>1 \mathrm{~m}$ \\
-Access control & $-\mathrm{HF}-13.5$ & $-\mathrm{UHF}-860 \mathrm{MHz}$ \\
-tracking & $\mathrm{MHz}$ & -Larger data \\
& & storage \\
& & -Backscatter \\
& & coupled \\
\hline
\end{tabular}




\section{Identification process}

\subsection{Communication Protocol}

A communications protocol defines therules of organizing the conversation between devices in the case of RFID, between tags and a reader to ensure that information actually gets transferred correctly and efficiently. A protocol defines, air interface which contains signal modulation, collision algorithm, request and response structure, data-coding. Information security.

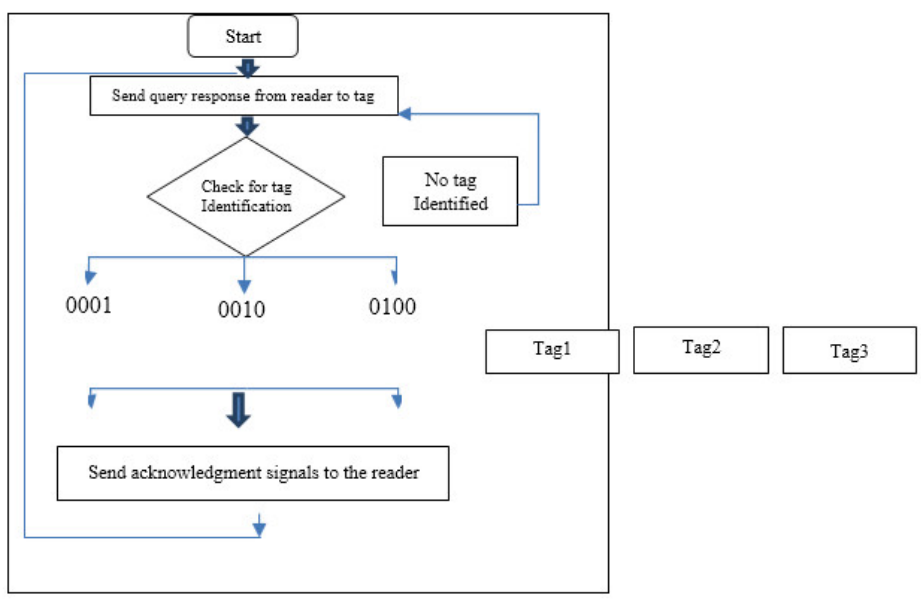

Figure. 3. Identification process

\subsection{RFID anti-collision algorithms :}

The anti-collision algorithm defines the way to identify multiple tags simultaneously with higher efficiency.First, we need to know the collisions categories: Tags Collisionshappen when multiple tags reply to a reader's request at the same time.Readers-to-tag Collisions happen when multiple readers readthe same tag, existing in their common range. Reader CollisionsHappen, when multiple readers are operatingat the same time, using the same frequency.

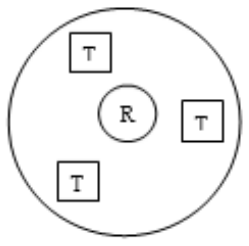

Figure. 4. Tags collision

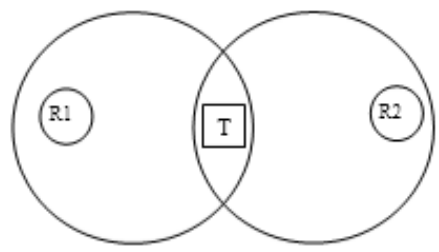

Figure. 5. Reader-to-Tag collision

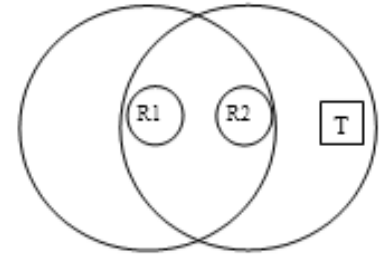

Figure.6. Readers collision

\subsubsection{ALOHA Algorithms:}

In RFID systems there are two most used anti-collision algorithms, ALOHA and Binary Tree algorithms based on Time Division Multiple Access (TDMA)which allows several users to share the same frequency channel by dividing the signal into different time slots.method. The reader request is divided into several slot. The reader can identify tag correctly when there is one tag responses in one slot. 
ForALOHAalgorithm,therearemanyadvancedversions, forexample,SlottedALOHA,FramedSlottedALOHAan dDynamicFramedSlottedALOHA.[5]

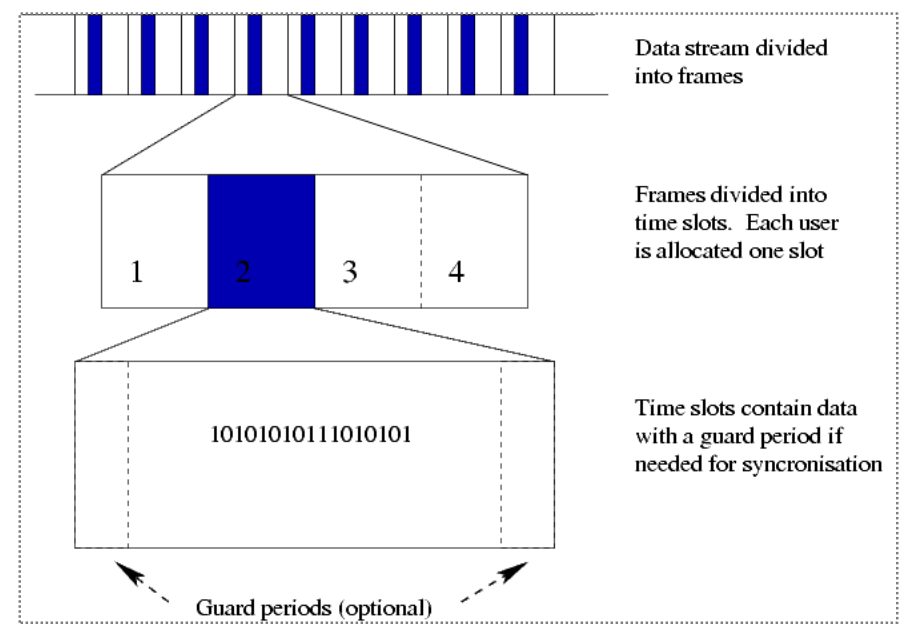

Figure. 7. TDMA frame structure

ALOHA algorithm is a simple anti-collision method based on TDMA. When the tag reaches the interrogation area of a reader, the tag will transmit the data immediately, and when more than one tag response at the same time, the collision occurs. So the most disadvantage of this algorithm is the high probability of collision.

In Slotted ALOHA algorithm, the time is divided into several slots, and the tag must transmit data in one slot, which it selects. So this method will decrease the probability of collision than ALOHA algorithm, but the reader and tag must communicate synchronously. When there is only one tag in one slot, reader can interrogate with tag and require the information of tag correctly. Due to the limitation of the number of slots, this algorithm used in the case that there are a few tags in the area.

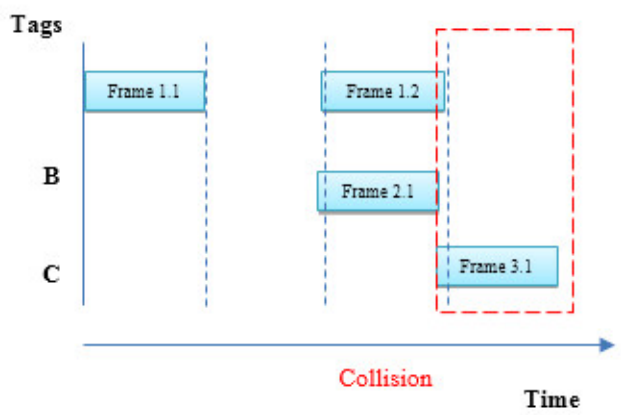

Figure. 8. Time divided into slots in slotted ALOHA

In Framed Slotted ALOHA (FSA) algorithm, one frame consists of several slots, and the tag will choose one slot in a frame to transmit data.FSA algorithm uses a fixed frame size and does not change the size during the process of tag identification. In FSA algorithm, the frame size is decided by the reader. Tags generate a random number that is used to select a slot in one frame and each tag then response in the slot it selected. Reader will identify tags with multiple frames, so it can solve the problem in slotted ALOHA algorithm. 
Since the frame size of FSA algorithm is fixed, its implementation is simple, but it has a weakness that drops efficiency of tag identification. For example, in FSA algorithm, when the number of tags is small, it should choose a small number of slots in one frame, or it will cause waste of empty slots; when the number of tags is large, it should choose a large number of slots in one frame, or there will be too many collisions and it will take a long time to identify all tags.

Dynamic Framed SlottedALOHA Algorithm has more advantages than other ALOHA-type algorithms, it is proposed to improve the performance of FSA algorithm, more efficient and most widely used.In DFSA algorithm, the reader can dynamically adjustthe frame size according to the number of existing unidentified tags,

At first, the reader sends a request command to the tags and indicates the frame size. In the first read cycle,tag 2 and tag3 transmit their data in slot3 simultaneously, and hence collision occurred. Tag1 and only tag1 sends itsdata in slot2, and hence the reader can successfully recognizetag1 because slot2 is singly occupied. Since there is only onetag recognizedinthe first read cycle,this implies there are still two tags that need to be recognized.Hence,the reader might determine a new contending frame size,three, for the next read cycle.

His process terminated until all tags were recognized or there is no collision occurring in one read cycle. In general, DFSA algorithm is more efficient than FSA algorithm because DFSA algorithm can dynamically adjust the contending frame size based on the current number of unidentified tags in the system.[6]

\subsubsection{Binary tree algorithm:}

TheBinaryTreealgorithmisbasedonatreemodel. It consists of many rounds. In each round, the reader transmits a query command and the tags respond with their IDs. The query command has a prefix. Only tags of which IDs match the prefix respond. When only one tag answers, the reader successfully recognizes the tag. When more than one tags answer, collision occurs and the reader can't recognize the tags. The reader, however, can know the existence of tags which IDs match the prefix. Then the reader tries to query with 1-bit longer prefixes in next rounds. By extending the prefixes, the reader can recognize all the tags.[5]

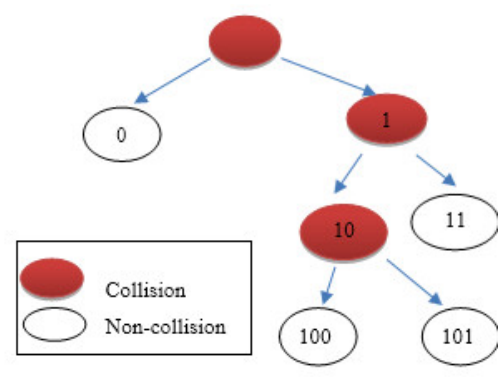

Figure. 9. Binary Tree algorithm

\subsection{RFID Protocols:}

There are many protocols about UHF RFIDfor exchanging information between a tag and a reader, such as EPC Class1 protocol, EPC Class1 Generation2 (Gen2), and ISO18000-6.

- EPC class1 protocol uses A bin-based Binary Tree algorithm, 
-EPCClass1Gen2 isastandardizationeffort,basedon DFSA Algorithm, proposed by EPC Global. It uses techniques like frequency hopping or frequency agile systems.

-ISO18000 - 6 type A protocol uses DFSA Algorithm

-ISO18000 - 6 type B protocol uses Binary Tree algorithm

\section{EPC Class1 Gen2}

Gen2 is an air interface protocol, which defines the physical and logical requirements for an RFID system of readers and passive tags, operating in the $860 \mathrm{MHz}-960 \mathrm{MHz}$ UHF range. It uses DFSA algorithm, and it is the most used.For Gen2 there is some operations used by reader to manage tag population, which are:Select, Inventory and Access. The reader uses "Select" to select tags population, and use "Inventory" to identify tags by transmitting a command in one of four sessions. One or more tags may reply. The reader detects a single tag reply and request the personal computer $(\mathrm{Pc})$. The process "access" is used when the reader reads tag or writes to individual tag, which should be uniquely identified prior to access [12].

\section{Conclusion}

To remote objects in the Internet of Things,each object is uniquely identified;weproposeRFID system to identify objects.RFID is a flexible, easy-to-use technology that is perfectly suited for automatic processing, it can also provide other services such as controlling lamps, a portal ... The software integrated in the reader can be customized according to the needs of the user.

The protocols and collision algorithms are existed to correctly identify things, to ensure securityand efficiency,DFSA algorithm and Gen2 protocol are the most used to avoid collision,organize communication between items, and to standardize the RFID operation.

\section{REFERENCES}

[1] Jae-Ryong Cha and Jae-Hyun Kim, "Dynamic framed slotted ALOHA algorithms using fast tag estimation method for RFID system," 2006, vol. 2, pp. 768-772.

[2] H. Safa, W. El-Hajj, and C. Meguerditchian, "A distributed multi-channel reader anti-collision algorithm for RFID environments," Comput. Commun., vol. 64, pp. 44-56, Jun. 2015.

[3] M. He et al., "A fast RFID tag identification algorithm based on counter and stack," Expert Syst. Appl., vol. 38, no. 6, pp. 6829-6838, Jun. 2011.

[4] Hamid Jabbar and Taikyeong Ted. Jeong, RFID System Integration. INTECH Open Access Publisher, 2010.

[5] I. SZENTMIKLÓSI and B. ILLÉS, "DESIGN, OPERATION AND ANTI-COLLISION PROCEDURE OF DATA TRANSFER OF IN ${ }^{\text {TEL }}$ LIGENT LOAD UNITS.”

[6] H.-W. Wang, "Efficient DFSA Algorithm in RFID Systems for the Internet of Things," Mob. Inf. Syst., vol. 2015, pp. 1-10, 2015. 
Douae Salim, Larbi Setti, Abdellatif Elabderrahmani, Identification of Objects by Machines using RFID Technology Identify Objetcs in Internet of Things. Transactions on Machine Learning and Artificial Intelligence, Vol 5 No 4 August (2017); p: 325-332

[7] A. Thomas, "RFID et nouvelles technologies de communication; enjeux économiques incontournables et problèmes d'éthique," in 6ème Conférence Internationale Conception et Production Integrées, CPI'2009, 2009.

[8] J. Myung, W. Lee, and J. Srivastava, "Adaptive binary splitting for efficient RFID tag anti-collision," IEEE Commun. Lett., vol. 10, no. 3, pp. 144-146, 2006.

[9] X. Yi, L. Wang, D. Mao, and Y. Zhan, "An Gen2 Based Security Authentication Protocol for RFID System," Phys. Procedia, vol. 24, pp. 1385-1391, 2012.

[10] http://www.enigmaticconsulting.com/Communications_articles/RFID/RFID_protocols.html

[11] https://www.etilux.be/fr/services/identification-etiquetage-marquage/tracabilite-rfid.php

[12] http://rfid4u.com/rfid-basics-resources/epc-gen2-reader-commands-and-q-parameter/ 